\title{
Transport and traffic management by micro simulation models: operational use and performance of roundabouts
}

\author{
F. G. Praticò ${ }^{1}$, R. Vaiana $^{2} \&$ V. Gallelli ${ }^{2}$ \\ ${ }^{1}$ University Mediterranea, Reggio Calabria, Italy \\ ${ }^{2}$ University of Calabria, Arcavacata Campus-Cosenza, Italy
}

\begin{abstract}
The performance of roundabouts can affect urban transport systems in terms of environmental and operational impacts, safety and efficiency. The development of roundabout traffic management and control systems can be carried out through road traffic micro-simulation models which are computer models where the movements of individual vehicles travelling around road networks are determined by using simple car following, lane changing and gap acceptance rules. Unfortunately, despite the great diffusion of these tools, appropriate methods are still needed in order to validate and calibrate these models. In general, the calibration process can be defined in this way: the process of comparing model parameters with real-world data to ensure that the model realistically represents the traffic environment. The objective is to minimize the discrepancy between model results and measurements or observations. The aim of this paper is the presentation of a first comparative approach between observed performances and performances obtained by the use of popular microsimulation software, in particular urban intersections such as roundabouts. In particular, an experimental investigation is designed and carried out in order to acquire some vehicular parameters for a roundabout placed in an urban contest of southern Italy. The calibration process is carried out by an analysis of variance of the kinematic parameters of an n-tuple of roundabout scenarios. This calibration procedure has permitted to derive some important conclusions about the choice of the most significant input parameters for the output results of each simulation scenario. Outcomes of this study are expected to benefit both practitioners and researchers.
\end{abstract}

Keywords: roundabout, micro-simulation models, calibration procedure, analysis of variance, kinematic parameters, car following. 


\section{Introduction}

Similar to traditional models, both statistical and analytical ones, which allow the evaluation of road junction performances (Capacity, Levels of Service, etc), today, the use of refined microscopic simulation techniques is more and more popular because they allow the study of road intersections, in particular roundabouts, through a dynamic approach.

Now different microsimulation software (such as SIDRA, CORSIM, VISSIM, PARAMICS, HCS, Synchro, RODEL, SimTraffic, etc) is used by practitioners and researchers. However it is important to underline that, despite the great diffusion of these instruments, there has not been the same improvement of appropriate methods in order to validate and calibrate these models.

In fact, microscopic simulation models contain many independent parameters to describe driver behaviour and traffic control operations. These models provide a default value for each parameter, but also allow users to change the values to represent local traffic conditions. However, along with a more and more refined analysis of microsimulation software algorithms, it is frequently necessary for each user (researcher, engineer, planner, etc.) to know the real sensitivity of these packages with regard to the most important key parameters.

In general, the calibrating process can be defined in this way [1]: The process of comparing model parameters with real-world data to ensure that the model realistically represents the traffic environment. The objective is to minimize the discrepancy between model results and measurements or observations.

\section{Organization of the paper}

The paper is organized in three steps as follows:

1) First, a brief discussion of previous studies related to the influence of kinematic and behavioural features in roundabouts microscopic simulation models is provided;

2) In the second part, the paper presents the first comparative approach between observed reality (OR) and simulated reality (SR), beginning from the acquisition of some principal vehicular parameters of a roundabout placed in a city in province of Cosenza (ITALY). In particular the main parameter considered for the comparison between OR-SR is the speed profile. The microsimulation process was conducted by the use of VISSIM ${ }^{\circledR} 4.1$ [2];

3) Finally, results and Analysis of Variance (ANOVA) of calibration ability are presented.

\section{Literature review}

Several software packages provide roundabout analysis capabilities, using various theoretical methods and requiring a variety of input parameters [3, 4]. However, only a few of these parameters usually have significant effects on 
analysis results. Therefore this part focuses on previous studies that examined the influence of these factors in different roundabout simulation models.

Gagnon et al. [5] presented a study where different roundabout models are considered: aaSIDRA, RODEL, PARAMICS, SimTraffic and VISSIM. In this paper, model evaluation was based on comparing approach delay values to actual field delays of two modern roundabout in New Hampshire (USA). The main conclusions of the research were the following: among the models considered, VISSIM appeared to be the most versatile, and RODEL seemed to be the least versatile; for aaSIDRA the Environment Factor (EF) appeared to have the most significant impact on the results, while for VISSIM, adjusting the minimum acceptable gap was a very powerful tool in calibrating the model; PARAMICS offered a number of calibrating factors, but, in this case, some of these parameters did not impact on the results.

The NCHRP [6] presented a study that compared capacity and delay estimates produced by RODEL and SIDRA in different roundabouts situated in the USA: 10 single-lane and 6 multilane sites. The analysis of results showed that, with queues under a minute, Rodel's delay was excessive, while SIDRA estimates were lower.

Bared and Edara [7], modeled two high-capacity roundabouts and their integration into smart signalized streams using VISSIM and then compared these simulation results with the results of RODEL and aaSIDRA. Finally, the comparison with real data collected from various sites in the USA showed that Vissim outputs were closer to real data than the RODEL and aaSIDRA results.

Kinzel and Trueblood [8] performed a sensitivity analysis for operational parameters related to roundabouts (such as critical gap, headway, follow-up time and speed) considering different models: HCS, Synchro, aaSIDRA, SimTraffic and VISSIM. This study, simulating hypothetical roundabout under three volume scenarios (balanced, unbalanced and congested), led to several conclusions: the parameters used in aaSIDRA (critical gap, follow-up headway, intra-bunch headway and $\mathrm{O} / \mathrm{D}$ factor) had a marked effect on the result; the effect of adjusting headway in SimTraffic was appreciable with lower-flow, but not dramatic, while, in the case of congestion, these effects were much more significant; in VISSIM, delay was much more sensitive to variations in the gapacceptance parameters.

The sensibility of several software is also discussed by Stanek and Milam [9]. They compared the capacity of roundabouts with flared entry and double lanes (a five-legged roundabout and a diamond interchange with roundabouts) obtained from RODEL, aaSIDRA, VISSIM and PARAMICS. The conclusions of this study pointed out that RODEL and aaSIDRA must be used to analyse highcapacity roundabouts only for unsaturated conditions or for isolated locations with standard geometry. PARAMICS and VISSIM instead should be used when oversaturated conditions are present in the study area or unique roadway geometry features are present.

Still considering the effect of operational parameters in the simulation of intersections, Park and Qi [10] carried out a study in which three microscopic traffic simulation models, VISSIM, PARAMICS and CORSIM, were selected 
for model review and practice of model calibration and validation. The simulation results were compared with the field data of two actuated signalized intersections in the USA to determine the performance of the calibrated models. The final considerations of this work were that different simulation models provide different sets of adjustable parameters and, generally, PARAMICS has the least calibration parameters, while CORSIM and VISSIM have more calibration parameters.

Therefore, after this brief digression, it is possible to conclude that, in order to obtain realistic output from the simulations, it is important to understand the definition and impact of each parameter. This study evaluates the effect of kinematic and behavioural parameters in the simulation of roundabouts by an analysis of variance (ANOVA), a statistical methodology that allows the evaluation of the influence of several control factors with different levels in a response variable [11].

\section{Experimental planning}

The experimental planning was organized in three steps (see figures 1 and 2 and tables 1-4):

a) Analysis of the geometric features of a geometrically regular roundabout. Main landscaping and design elements are defined and summarized in the following table 1. In connection with table 1 some definitions are needed: Entry/Exit Radius: the minimum radius of curvature of the outside or right curb at the Entry/Exit | Entry/Exit Width: the width of the Entry/Exit where it meets the inscribed circle, measured perpendicularly from the right edge of the entry to the intersection point of the left edge line and the inscribed circle $\mid$ Inscribed Circle: the circle forming the outer edge of the circulatory roadway used to define the size of a roundabout. Central Island: the raised area in the center of a roundabout around which traffic circulates | Splitter Island: a raised or painted area on an approach (it separates/deflects/slows traffic and provides storage space for pedestrians) | Circulatory Roadway Width: the width between the outer flow line of the circulatory roadway and the central island. It does not include the width of any apron.

b) Vehicular flow data collection and micro-simulator input data processing;

c) Choice of variables and implementation of scenarios by micro-simulation software.

The four-leg roundabout studied in this paper is located in the University Campus of Arcavacata, in the province of Cosenza (ITALY). This intersection allows a continuous flow of traffic through the use of the yield and merge manoeuvres (Figure 1). 
Table 1: $\quad$ Roundabout geometric properties.

\begin{tabular}{|c|c|c|c|c|c|c|}
\hline \multirow{6}{*}{ 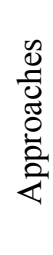 } & \multirow{2}{*}{ ID } & \multicolumn{2}{|c|}{ Entry $(\mathrm{m})$} & \multicolumn{2}{|c|}{ Exit (m) } & \multirow{2}{*}{$\begin{array}{c}\text { Splitter Island } \\
\text { Width (m) }\end{array}$} \\
\hline & & Radius & Width & Radius & Width & \\
\hline & $\mathrm{A}$ & 8.00 & 4.22 & 28.32 & 4.31 & 2.80 \\
\hline & $\mathrm{B}$ & 14.20 & 4.13 & 8.00 & 4.33 & 2.20 \\
\hline & $\mathrm{C}$ & 5.65 & 5.01 & 14.20 & 4.78 & 2.40 \\
\hline & $\mathrm{D}$ & 28.32 & 5.38 & 5.65 & 5.58 & 3.00 \\
\hline \multicolumn{6}{|c|}{ Radius Central Island (m) } & 4.25 \\
\hline \multicolumn{6}{|c|}{ Inscribed Circle Diameter (m) } & 23.00 \\
\hline \multirow{2}{*}{\multicolumn{4}{|c|}{ Circulatory Roadway }} & \multicolumn{2}{|c|}{ Width (m) } & 6.00 \\
\hline & & & & \multicolumn{2}{|c|}{ Traversable Apron (m) } & 1.25 \\
\hline
\end{tabular}

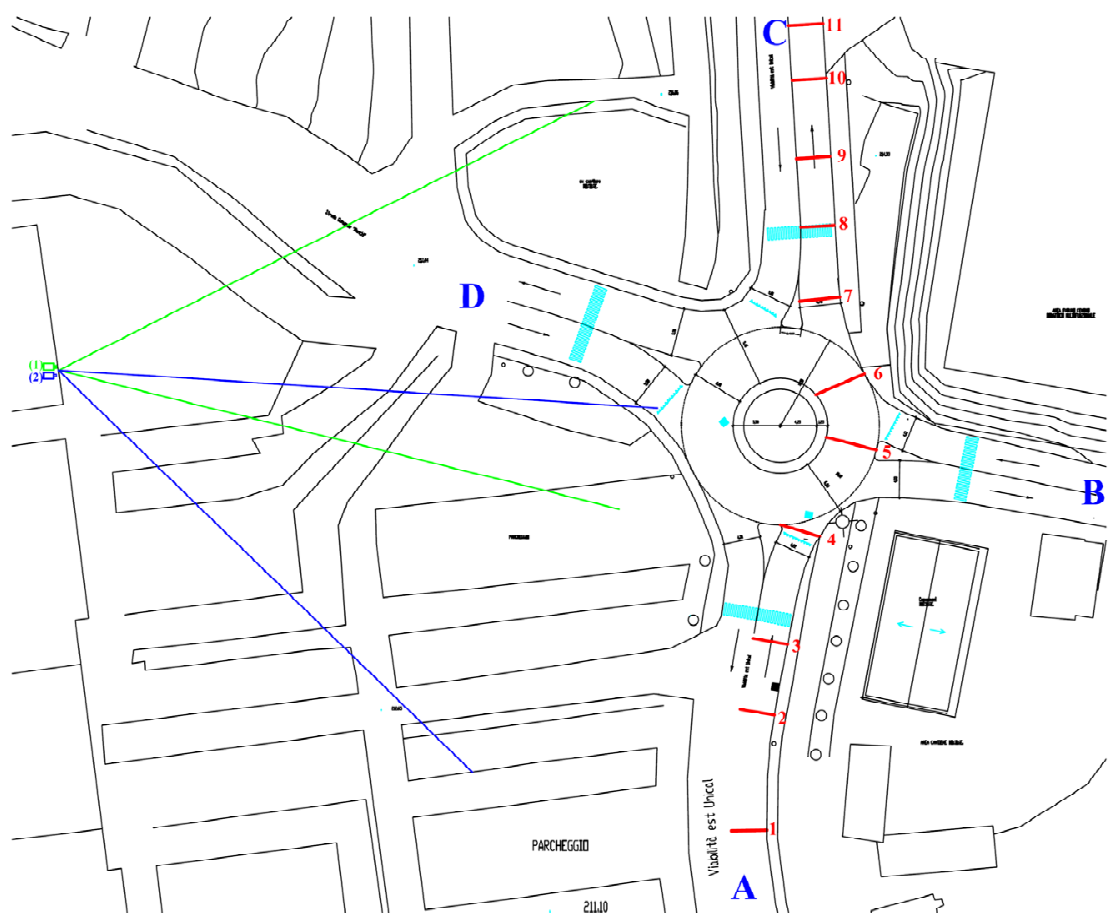

Figure 1: Roundabout plant (note the two points of shot and the position of the sections).

\subsection{Data collection}

The roundabout was video-taped by two cameras: the point of shot is shown in figure 1. This intersection was recorded from 7:30 am to 2:30 pm. Historically, 
the peak volumes occurred in the morning from 8:00 am to 9:00 am and in the afternoon from 1:00 pm to 2:00 pm (Saturation Flow Configuration, SFC).

From the video-tapes we derived traffic flows, the time of service for each entry, the drivers' headways and acceptable gaps (during peak hours), speed profiles (with respect to the lowest traffic period that occurred from 10:00 am to 12:00 pm - Free Flow Configuration, FFC). All the information was collected for 1-minute time increments. The Origin-Destination (OD) matrixes (homogenized in vehicle per hour, (1), are summarized in table 2.

Table 2: Volume of traffic for Free Flow and Saturation Flow Configuration.

\begin{tabular}{|c|c|c|c|c|}
\hline Min-Av-Max (vph) & A & $\mathrm{B}$ & $\mathrm{C}$ & $\mathrm{D}$ \\
\hline $\mathrm{A}(\mathrm{FFC})$ & $0-13-120$ & $0-45-180$ & $0-194-450$ & \begin{tabular}{|l}
$60-208-540$ \\
\end{tabular} \\
\hline $\mathrm{B}(\mathrm{FFC})$ & $0-40-180$ & 0 & $0-4-60$ & $0-55-240$ \\
\hline $\mathrm{C}(\mathrm{FFC})$ & $0-117-300$ & $0-4-60$ & 0 & $0-42-180$ \\
\hline $\mathrm{D}(\mathrm{FFC})$ & $60-143-420$ & $0-38-180$ & $0-32-240$ & $0-4-60$ \\
\hline $\mathrm{A}(\mathrm{SFC})$ & 0 & $0-184-420$ & $300-650-1080$ & $60-278-720$ \\
\hline $\mathrm{B}(\mathrm{SFC})$ & $120-377-840$ & 0 & $0-84-300$ & $0-104-360$ \\
\hline $\mathrm{C}(\mathrm{SFC})$ & $120-426-960$ & $0-80-240$ & $0-36-180$ & $0-65-300$ \\
\hline $\mathrm{D}(\mathrm{SFC})$ & $240-551-1080$ & $0-171-420$ & $0-42-240$ & $0-1-60$ \\
\hline
\end{tabular}

As is well known, the Time of Service is the time that a driver spends at the top of the queue before he can "make use" of the roundabout [12].

All the times of service were collected per 1-minute time increments for each approach during the Saturation Flow Configuration and the average time of service was associated to each 1-minute period [13]. The following exponential relationship between the time of service (Y) and the circulating flow, $\mathrm{x}$, was obtained, see $[13,14]$.

$$
\mathrm{Y}=1.93 \cdot \exp (0.03 \cdot \mathrm{x}) \quad\left(\mathrm{R}^{2}=0.88\right)
$$

The acceptable gap is the time-gap between two consecutive vehicles in the circulating roadway accepted by a driver placed at the entry of the roundabout so that he can make his movement in safe conditions [12]).

Acceptable gaps were collected during the peak periods (1397 admissions in roundabout were analysed). A log-normal distribution was obtained, its probability density function resulting:

$$
N=\frac{1}{A \cdot \sigma \cdot \sqrt[2]{2 \pi}} \cdot e^{-\left(\frac{(\ln A-\mu)^{2}}{2 \cdot \sigma^{2}}\right)}
$$


where $\mathrm{N}$ is the probability density function corresponding to A (acceptable gap), while $\mu$ and $\sigma$ are the mean and standard deviation, respectively, of the variable's natural logarithm. In more detail, the range $2.0-4.5$ s represented about $50 \%$ of all acceptable gaps.

From the analysis of the tapes during the lowest traffic period helpful information were obtained about the speed distribution. As for the through movement A-C the operating speed (V85) resulted a convex function (no line segment lies below the V85 graph at any point). For distances from $40 \mathrm{~m}$ to $15 \mathrm{~m}$ from the middle of the splitter island of the approach B, V85 decreased from around $35 \mathrm{~km} / \mathrm{h}$ to around $20 \mathrm{~km} / \mathrm{h}$. In contrast, in the range $5-45 \mathrm{~m}$ (after the middle of the splitter island of the approach B), V85 increased from around $20 \mathrm{~km} / \mathrm{h}$ to around $45 \mathrm{~km} / \mathrm{h}$.

\subsection{Setting up of scenarios}

The Data collected were used to investigate the calibration of VISSIM microsimulation model, under FFC conditions.

The following parameters were considered:

- $\quad$ O/D matrixes collected during the lowest traffic periods (FFC);

- choice of speed distribution for approach speed, reduced speed area, circulatory speed and exiting speed still considering the FFC;

- definition of minimum gap and headway of the priority rules starting from the data collected during the SFC;

- $\quad$ as for driver behaviour elements, the parameters detailed in table 3 were used (cfr. Wiedemann [2]).

Table 3: Default parameters of Wiedemann '74 model used in Vissim.

\begin{tabular}{|c|c|c|c|c|}
\hline \multirow{4}{*}{\multicolumn{2}{|c|}{$\begin{array}{c}\text { Car-following } \\
\text { model }\end{array}$}} & \multicolumn{2}{|c|}{ Look ahead distance } & $0.00 \mathrm{~m}-250.00 \mathrm{~m}$ \\
\hline & & \multicolumn{2}{|c|}{ Average standstill distance } & $2.00 \mathrm{~m}$ \\
\hline & & \multicolumn{2}{|c|}{ Additive part of desired safety distance } & 2.00 \\
\hline & & \multicolumn{2}{|c|}{ Multiple part of desired safety distance } & 3.00 \\
\hline \multirow{3}{*}{$\begin{array}{c}\text { Lane } \\
\text { Change }\end{array}$} & \multirow{2}{*}{\multicolumn{2}{|c|}{ Max Deceleration }} & Own & Trailing Vehicle \\
\hline & & & $-4.00 \mathrm{~m} / \mathrm{s}^{2}$ & $-3.00 \mathrm{~m} / \mathrm{s}^{2}$ \\
\hline & \multicolumn{2}{|c|}{ Accepted Deceleration } & $-1.00 \mathrm{~m} / \mathrm{s}^{2}$ & $-1.00 \mathrm{~m} / \mathrm{s}^{2}$ \\
\hline \multicolumn{3}{|c|}{ General behaviour } & \multicolumn{2}{|c|}{ Free lane selection } \\
\hline \multicolumn{3}{|c|}{ Lateral behaviour } & \multicolumn{2}{|c|}{ Desired position at free flow: middle of lane } \\
\hline
\end{tabular}

The variables for the setting up of scenarios were chosen through a careful analysis about the most significant input parameters for the variation of output results [3].

In total, 216 scenarios for single-lane roundabouts (DS Scenarios = Desired Speed sections in the circulating lane) were composed and analysed. In Table 4 input data are summarized. 
Table 4: $\quad$ Summary of input data.

\begin{tabular}{|c|c|c|}
\hline Type & Parameters & Input Data \\
\hline Fixed value & Traffic Flow & O/D matrix collected during FFC \\
\hline Variables & $\begin{array}{c}\text { Desired Approach } \\
\text { Speed }\end{array}$ & $\begin{array}{r}\mathrm{S}_{1}=30-55 \mathrm{~km} / \mathrm{h} ; \mathrm{S}_{2}=25-50 \mathrm{~km} / \mathrm{h} ; \\
\mathrm{S}_{3}=20-45 \mathrm{~km} / \mathrm{h} .\end{array}$ \\
\hline Variables & $\begin{array}{c}\text { Length of reduced } \\
\text { speed area }\end{array}$ & $\begin{array}{c}\mathrm{L}_{1}=0 \mathrm{~m} ; \mathrm{L}_{2}=2 \mathrm{~m} ; \mathrm{L}_{3}=6 \mathrm{~m} ; \mathrm{L}_{4}=8 \mathrm{~m} ; \\
\mathrm{L}_{5}=10 \mathrm{~m} ; \mathrm{L}_{6}=12 \mathrm{~m} .\end{array}$ \\
\hline Variables & $\begin{array}{c}\text { Position of Desired } \\
\text { Speed at Exit }\end{array}$ & $\mathrm{EX}_{1}=0 \mathrm{~m} ; \mathrm{EX}_{2}=6 \mathrm{~m} ; \mathrm{EX}_{3}=12 \mathrm{~m}$. \\
\hline Variables & Time Gap & $\begin{array}{c}\mathrm{G}_{1}=2.5 \mathrm{~s} ; \mathrm{G}_{2}=3.0 \mathrm{~s} ; \mathrm{G}_{3}=3.5 \mathrm{~s} ; \\
\mathrm{G}_{4}=4.0 \mathrm{~s} ; \mathrm{Headway}=5 \mathrm{~m}\end{array}$ \\
\hline Fixed value & $\begin{array}{c}\text { Desired Speed range in } \\
\text { the circulatory roadway }\end{array}$ & $\mathrm{S}=10-25 \mathrm{~km} / \mathrm{h}$. \\
\hline Fixed value & $\begin{array}{c}\text { Speed range in the } \\
\text { reduction speed area }\end{array}$ & $\mathrm{S}=15-25 \mathrm{~km} / \mathrm{h}$. \\
\hline
\end{tabular}

\section{Results and analyses}

By referring to the through movement A-C, the simulation results were analysed in terms of average speed for the through movement and compared with the experimental data collected along the same path during the lowest traffic period. Therefore it was possible to derive an average per cent error for each simulation and to perform an Analysis of Variance (ANOVA).

The average per cent error, Er\% was derived as follows:

$$
E r \%=\frac{1}{N} \cdot \sum_{i=1}^{N} \frac{\left|S_{S R_{i}}-S_{O R_{i}}\right|}{S_{O R_{i}}} \cdot 100 \quad \text { with } \mathrm{i}=1, \ldots ., 10
$$

where $\mathrm{S}_{\mathrm{SRi}}$ is the speed measured on the section "i" of the Simulated Reality $(\mathrm{SR}), \mathrm{S}_{\mathrm{ORi}}$ is the speed measured on the section "i" of the Observed Reality (OR), $\mathrm{N}$ is the number of Speed Sections.

Due to the stochastic nature of VISSIM's simulation model ("Random Seed"), it was necessary to run each scenario many times and to average the results in order to provide a 95\% confidence (confidence interval of $\pm 0.50 \mathrm{Km} / \mathrm{h}$ ). Average speed and standard deviation (deriving from the average of the 10 simulations for each scenario) for the best and worst DS scenarios are reported in the following figures.

Furthermore, an Analysis of Variance (ANOVA) was performed. The following variables were considered with respect to the through movement A-C: i) critical gap [Gi]; ii) length of reduced speed area [Li]; iii-iv) position of desired exiting speed section [Exi] and approach speed [Si], see figure 3 .

For each variable the following indicators were derived: DF (degree of freedom); Seq SS (sequential sum of squares); Adj SS (adjusted sum of squares); 

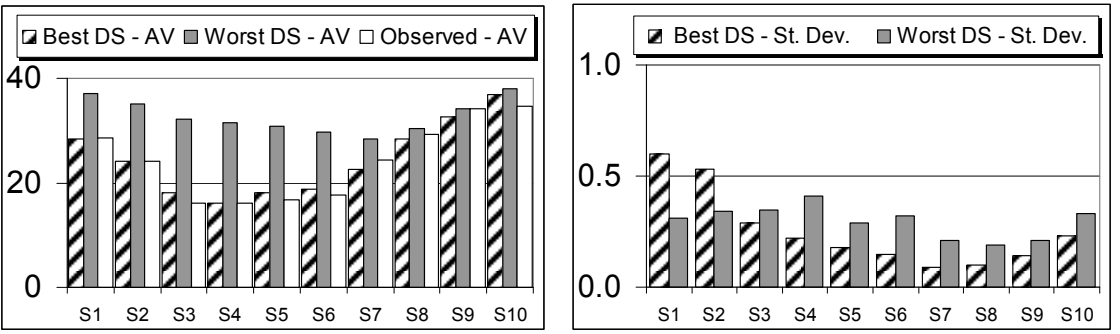

Figure 2: $\quad$ Average speed (AV) and standard deviation (ST DEV), Km/h, for the best and worst DS scenarios and observed speed along the through movement A-C, for each section S1-S10.

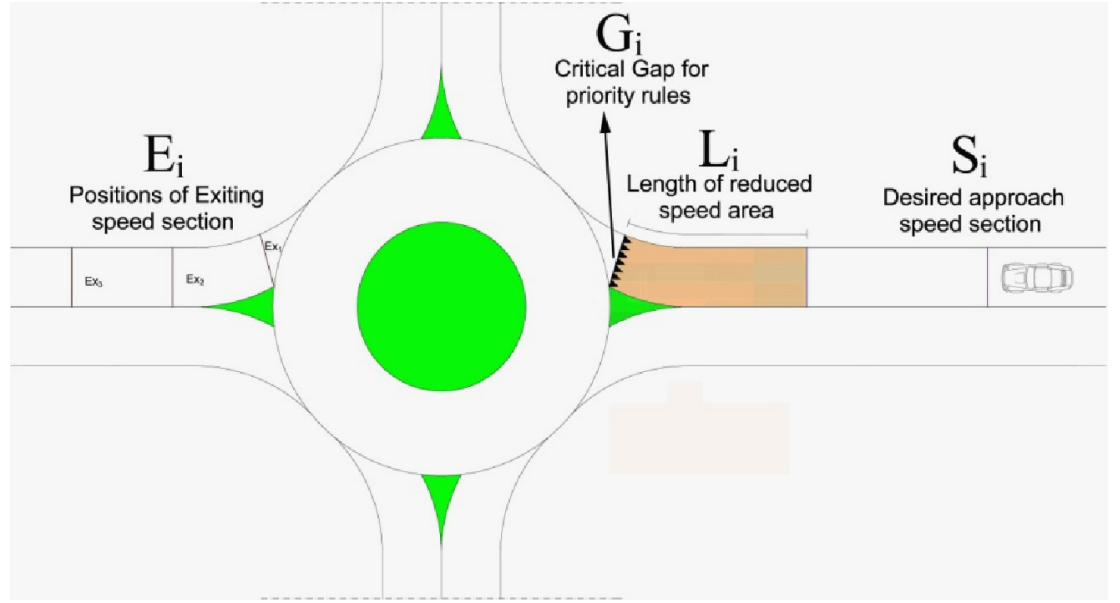

Figure 3: Variables considered with respect to the through movement A-C in the Analysis of Variance.

Adj MS (adjusted mean square); F (F-statistic); P (p-values). Based on the results, all the four factors and only two interactions ( $\mathrm{Gi}^{*} \mathrm{Li}$ and $\mathrm{Exi}{ }^{*} \mathrm{Si}$ ) were significant [15]).

For each considered factor (x-axis: critical gap, Gi; length of reduced speed area, Li; position of desired exiting speed section, Exi; desired approach speed, $\mathrm{Si})$, the average per cent error between simulated speed and observed speed along the through movement A-C (y-axis) was derived (the so-called main effects plot were used). Furthermore, in the aim of analysing the effects on the data of any interaction between conditions we used the interaction plots (x-axis: a given variable among the four above mentioned; $y$-axis: average per cent error; parameter: another parameter among the abovementioned four ones).

As a consequence, for each parameter we derived the following best-fit values: i) Critical Gap: $3.5 \mathrm{~s}-4.0 \mathrm{~s}$; ii) length of reduced speed zone: between $6 \mathrm{~m}$ 
and $8 \mathrm{~m}$; iii) position of the desired exiting speed section: immediately after the exit from the roundabout next to the top of the splitter island; iv) range of approach speed: $25-50 \mathrm{Km} / \mathrm{h}$.

Furthermore, by referring to the speed profiles for the through movement A$\mathrm{C}$, it is noted that the best RZ scenario (where the use of the reduced speed zone was assumed) yielded results similar to the average speeds observed for distances lower than $-5 \mathrm{~m}$, while the best DS scenario (where the use of the reduced speed zone was not assumed) better fitted observed speeds.

Based on the analysis of micro-simulated speeds, it is possible to observe that the use of reduced speed zones in the circulating lane did not perform a satisfactory simulation of observations as in literature [17] and the prediction of operating speeds still call for further research [18].

\section{Conclusions}

Traffic simulation models and software have become more and more popular as a traffic analysis tool used in transportation analyses.

Indeed, there is the need to model and analyse the operation of complex transportation systems under normal or congested conditions.

However, despite the extensive use of traffic simulation software, there are a variety of conflicting opinions on how simulation should be calibrated and used. In more detail, the calibration procedure emerges as strategic for an accurate use of micro-simulation software.

The use of an indicator which measures the percentage difference between OR (observed reality) and SR (simulated reality) configuration allowed us to evaluate the sensitivity of micro-simulation outputs to several input parameters. Authors are aware of the fact that the obtained results cannot be extended to general cases.

On the other hand, it is well known that calibration procedures need a preliminary analysis of the main factors and variables (approach speed, acceptable gaps, time of service, O/D matrixes, etc.). Indeed, they represent the basic element from which to start a correct setting of the scenarios.

The calibration procedure carried out has allowed us to draw some preliminary conclusions about the choice of the most significant input parameters.

For the case-study under investigation, the optimal critical gap ranged from $3.5 \mathrm{~s}$ to $4.0 \mathrm{~s}$, while the optimal length of the reduced speed zone for each entry was at least 6-8 $\mathrm{m}$, in order to obtain an accurate speed profile for the through movement. Furthermore, the best position of the desired exiting speed section was the one immediately after the exit from the circulatory roadway and the use of reduced speed zones in the circulating lane did not perform a satisfactory simulation of observations.

Outcomes of this study are expected to benefit both practitioners and researchers.

Further research will be needed to complete the study aimed at gaining a more comprehensive understanding of input criticality. 


\section{References}

[1] HCM. Highway Capacity Manual. Transportation Research Board, Washington, 2000.

[2] VISSIM Manual User - Release 4.10. PTV Planung Transport Verkehr, Karlsruhe, Germany, 2005.

[3] Gallelli, V., Vaiana, R., Roundabout intersections: evaluation of geometric and behavioural features with VISSIM. Proceedings of TRB - National Roundabout Conference, Kansas City, Missouri, 2008.

[4] Vaiana, R., Gallelli, V., Capiluppi, G.F., Roundabout intersections: analysis for scenarios by micro-simulation. Proceedings of 4th International SIIV Congress - Advances in transport infrastructures and stakeholder expectations, Management Session + Poster Session, Palermo, 2007.

[5] Gagnon, C., Sadek, A., W. Touchette, A., Smith, M., Calibration potential of common analytical and micro-simulation roundabout models: a New England case study. Proceedings of TRB of the National Academies, Washington, D.C., 2008.

[6] Rodegerdts, L. Applying Roundabouts in the United States. Publication NCHRP 3-65, Committee on Highway Capacity and Quality of Service, Washington, D.C., 2004.

[7] Bared, J., Edara, P.K., Simulated Capacity of Roundabouts and Impact of Roundabout Within a Progressed Signalized Road. Proceedings of TRB National Roundabout Conference, Vail, Colorado, 2005.

[8] Kinzel, C. Trueblood, M. The Effects of Operational Parameters in the Simulation of Roundabouts. Kansas City, Missouri, 2004.

[9] Stanek, D., Milam, R., High-capacity roundabout intersection analysis: going around in circles. Proceedings of TRB - National Roundabout Conference, Vail, Colorado, 2005.

[10] Park, B., Qi, H., Development and evaluation of a calibration and validation procedure for microscopic simulation models. Final Contract Report VRTC 05-CR1, Virginia Transportation Research Council, Charlottesville, Virginia, 2004.

[11] Montgomery, D.C., Design and Analysis of Experiments, Wiley and Sons INC: New York, 1997.

[12] Mauro, R., Calculation of roundabouts, Springer: Berlin-Heidelberg, 2010.

[13] Capiluppi, G. F., Gallelli, V., Vaiana, R., Intersezioni a raso con soluzione a rotatoria dissimmetrica: un caso di studio. Proceedings of 16th National SIIV Congress, Rende, Italy, 2006.

[14] Capiluppi, G. F., Gallelli, V., Vaiana, R., La microsimulazione del traffico veicolare in rotatoria. Applicazione ad un caso reale. Proceedings of 18th National SIIV Congress, Enna, Italy, 2008.

[15] Cunto, F. and Saccomanno, F., Calibration and validation of simulated vehicle safety performance at signalized intersections. Accident analysis \& prevention, 2008.

[16] Trueblood, M. Dale, J. Simulating roundabouts with Vissim. Kansas City, Missouri, 2004. 
[17] U.S. Department Of Transportation. Roundabouts: an informational guide. Report n. FHWA-RD-00-067, Washington D.C., 2000.

[18] Praticò F. G, Giunta M. S., Speed distribution in low volume roads: from inferences to rehabilitation design criteria, "Transportation research record”, n. 2203, 2011, ISSN: 0361-1981. 